\title{
ANALYSIS AND IMPLEMENTATION OF AN ORBITAL ORIENTATION CONTROLSYSTEM FOR A PICO-SATELLITE
}

\author{
MIGUEL A. ÁVILA A, JULIÁN R. CAMARGO L \& OSCAR D. FLÓREZ C \\ Engineering Faculty, Universidad Distrital Francisco José de Caldas, Bogotá, Colombia
}

\begin{abstract}
This project presents the development of a stabilization and orientation system for the space vehicle in orbit that allows obtaining the adequate orientation to establish communication with the ground station. This requirement is achieved through the implementation of a joint actuator and sensor system for the acquisition, reacquisition and reorientation of the satellite. Taking into account that the proposal establishes the use of a triaxial gyroscope as an actuator, as well as the implementation of solar sensors located in each of the faces of the pico-satellite. Allowing fast actions and the use of little memory in the main program.
\end{abstract}

KEYWORDS: Orientation, Attitude Determination, Actuator, Sensor \&Orbital Disturbances

Received: Jun 08, 2020; Accepted: Jun 28, 2020; Published: Sep 17, 2020; Paper Id.: IJMPERDJUN20201300

\section{INTRODUCTION}

This work shows the analysis and implementation of an orbital orientation system, known as attitude determination and control system of a satellite are formalized to determine the spatial orientation in its orbital trajectory, for which it is required the re-relationships between the various rectangular coordinate systems located both in the primary body as well as the coordinate systems in the space vehicle namely, sidereal, perifocal, topocentric, spacecraft body, main spacecraft, spacecraft orbit [1].

The project due to its size and mass limitation $1000 \mathrm{~g}$ allows only the implementation of a passive actuator, which for our case is a magnetometer that acts as an actuator and the use of solar sensors to obtain a measurement of the orientation angle, which will allow taking the appropriate decisions to send them to the satellite and that the actuator performs the relevant attitude action, to have an adequate orientation to ensure the realization of the link with the ground station and go to the mission status.

The proposed implementation of the attitude system is a triaxial gyroscope, which will always be in the disposition of aligning with the Earth's magnetic field, for which a sensor measurement will be available that must be equal to the attitude of the satellite minus the nominal attitude of the simulation calculations.

\section{ORIENTATION AND ATTITUDE OF THE SATELLITE}

A space vehicle in orbit is subject to the gravitational actions of the Earth and the perturbations due to the space environment, so this term in aerospace engineering refers to the angular orientation of a fixed coordinate system on the satellite concerning to the external coordinate system. [2]

This means that the movement of the satellite in space can be described by a set of equations that are of the ordinary non-linear differential type, whose solution allows the knowledge of the external torque, angular momentum 
and of course its attitude to a reference coordinate system, which allows the use of translation and rotation equations between the systems.

The spacecraft attitude can be understood as the process of measuring the angular orientation of the satellite through sensors. Then the term attitude acquisition is used for a maneuver in which the initial attitude is unknown at the time of planning the action [3].

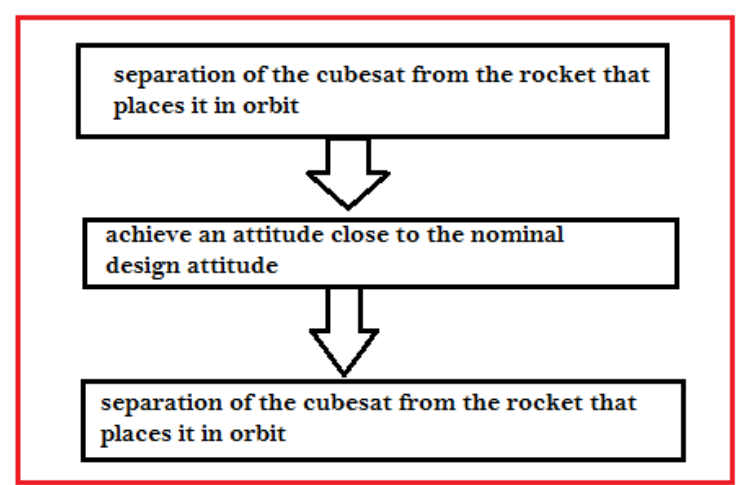

Figure 1: Steps to Obtain a Satellite Attitude Acquisition.

Thus, the movement of the satellite in its trajectory can be studied from two points of view according to the variables that are present to know; a kinematic study, which relates the orbital speed, its relative orientation that involves the knowledge of the angles of Euler, its location for the primary body, although we handle three variables we need four that we handle them with the unitary quaternion $\mathrm{q}$ (the combination of three components of the unit vector and the angle of rotation). [4].

From the dynamic point of view, the pairs of external forces acting on the system are studied, such as the perturbations of the space environment (gravitational, friction, solar radiation and magnetic pairs), and those of the control system[5].

\subsection{Representation of Vehicle Orientation in Orbit}

In a satellite project, there is a fundamental problem such as the representation of the attitude of the space vehicle in orbit, which represents the torques exerted on the artificial ship and the temporal evolution of its orientation, this is resolved by thinking of being able to obtain the orientation of the coordinate system tied to the body of the satellite concerning a fixed coordinate system in the primary. [6]

The known proposals to find the solution to this problem are the Euler angles, the unit quaternion and the $\mathrm{R}_{313}$ rotation matrix.

\subsection{Reference Systems}

The coordinate systems can be differentiated in terms of the location of their origin, they can be located in the primary body and the spacecraft, the definition of these reference systems must take into account the geometric conditions (mandatory and linked to the geometric shape of the satellite) and physical conditions (they are external, not fixed to the rotating body and not necessarily inertial) these correspond to [7].

- Earth's coordinate system ECEF: Assumes a length of zero at the Greenwich meridian, used to enter fixed coordinates in the ship. [10]. 
- Main in body (SCB): its geometrical axis is fixed, but its origin is placed in the center of the satellite mass.

- Main in Orbit (SCO): its axes are parallel to the ECI, but with origin in the center of mass of the space vehicle.

- Main vehicle (SCP): its origin is placed in the center of the satellite mass. [10]

- Earth Inertial System ECI has the origin of the system in the Earth's center of mass, being its Z-axis parallel to the Earth's axis of rotation. [10]

\section{DYNAMIC MODELLING}

It is the grouping of the equations of kinematics and dynamics, for it you can use methods of numerical or analytical models, starting from: [11]

$$
\vec{\tau}=\frac{d \vec{L}}{d t}
$$

The state vector has to be defined from the angular velocity vectors $\omega$ and the angular momentum $\mathrm{L}$ parameterized and selected for the ADACS, as well as the degrees of freedom. If the internal torque is zero for the ship, the expression for the net external torque corresponds to: [8]

$$
\begin{aligned}
& \vec{\tau}=\vec{\omega} X \vec{L}+\frac{d \vec{L}}{d t} \\
& \vec{\tau}=\left(I^{-1} \vec{L}\right) X \vec{L}+\frac{d \vec{L}}{d t}
\end{aligned}
$$

But if the internal torque is different from zero, the sum of the torques for the reference system in the ship is taken:

$$
\vec{\tau}=I^{-1}(\vec{L}-\vec{h}) X \vec{L}+I \frac{d \vec{L}}{d t}
$$

An expression containing ( $\mathrm{g}$ the internal torque exerted on the space vehicle. In this way, we can represent the kinetic energy of the system in the form:

$$
E_{C r}=\frac{1}{2} I \omega^{2}
$$

\section{GYROSCOPIC STABILITY}

A gyroscope maintains an angular reference direction through a mass with an attached spin. The use of this instrument requires consideration:

- If the mass $m$ is very small, the gyro can be used as a sensor.

- The modeled rotation consists of using sensors to sense the angular velocity $\omega$ to integrate the equations of the kinematics.

- Parallel component: causes an acceleration.

- If the mass is very large, use the gyro as an actuator.

- It should be noted that the space environment exerts a torque $\tau$ on the space vehicle which has components that perform the following actions: 
Normal component: causes a displacement of Lin the direction of the applied torque $\tau$.Euler's equations establish:

$$
\vec{\tau}=\frac{d \vec{L}}{d t}
$$

If the angular momentum $h$ of the vessel is taken into account, the external torque on the vehicle has a normal component at $h$. A differential displacement will then be obtained which can be calculated by:

$$
\Delta \theta=\frac{F r}{I \omega}
$$

Letting us see that the higher $\mathrm{h}$ the lower $\Delta \theta$, a condition known as gyroscopic stability. [11]

\section{PASSIVE CONTROL}

It uses principles of forces or torques exerted in the space environment. It presents some advantages as saving onboard resources (saving battery power) but has a disadvantage as providing poor control and little precision. The project proposes the use of fixed rotors to the structure of the ship by transferring an $L$ when rotating [16].

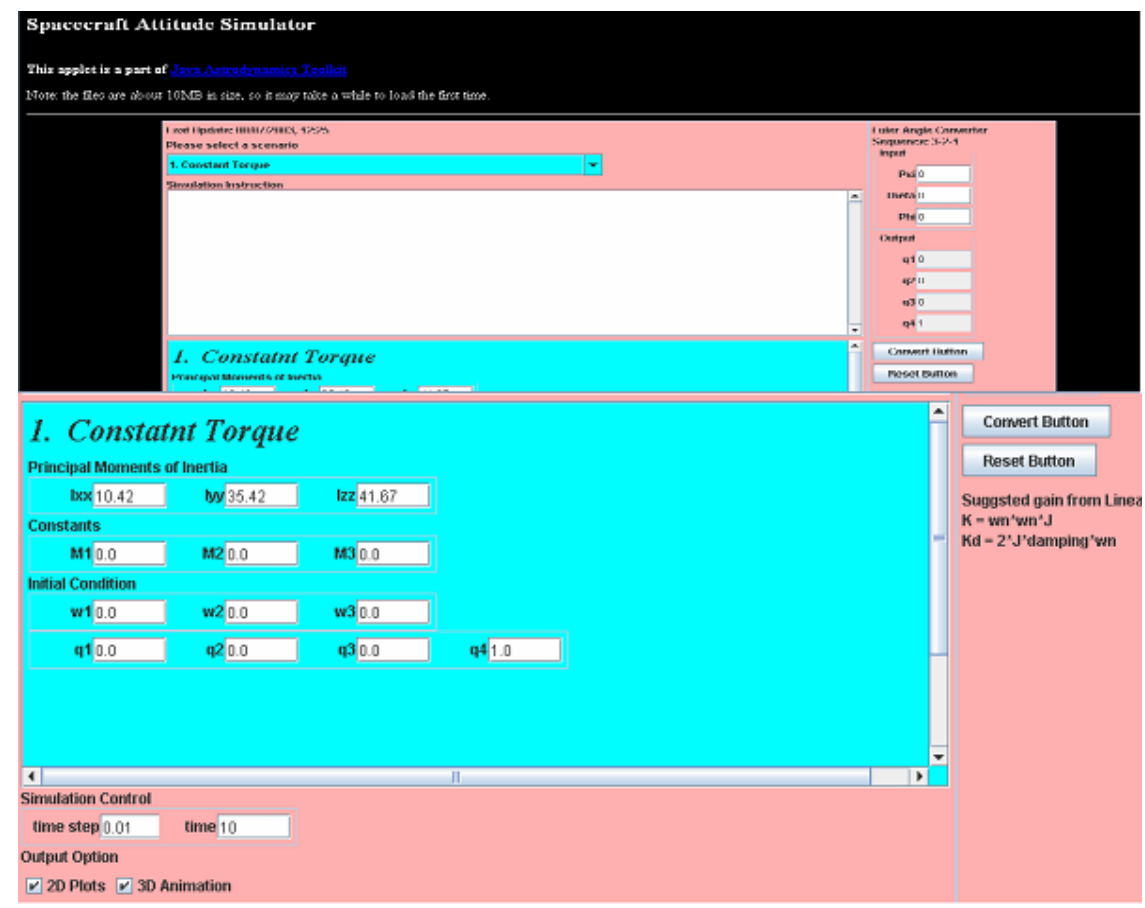

Figure 2: Simulation Data in Attitude Spacecraft Simulator.

\section{SIMULATION}

Below are some figures that have been obtained in the simulation process: 

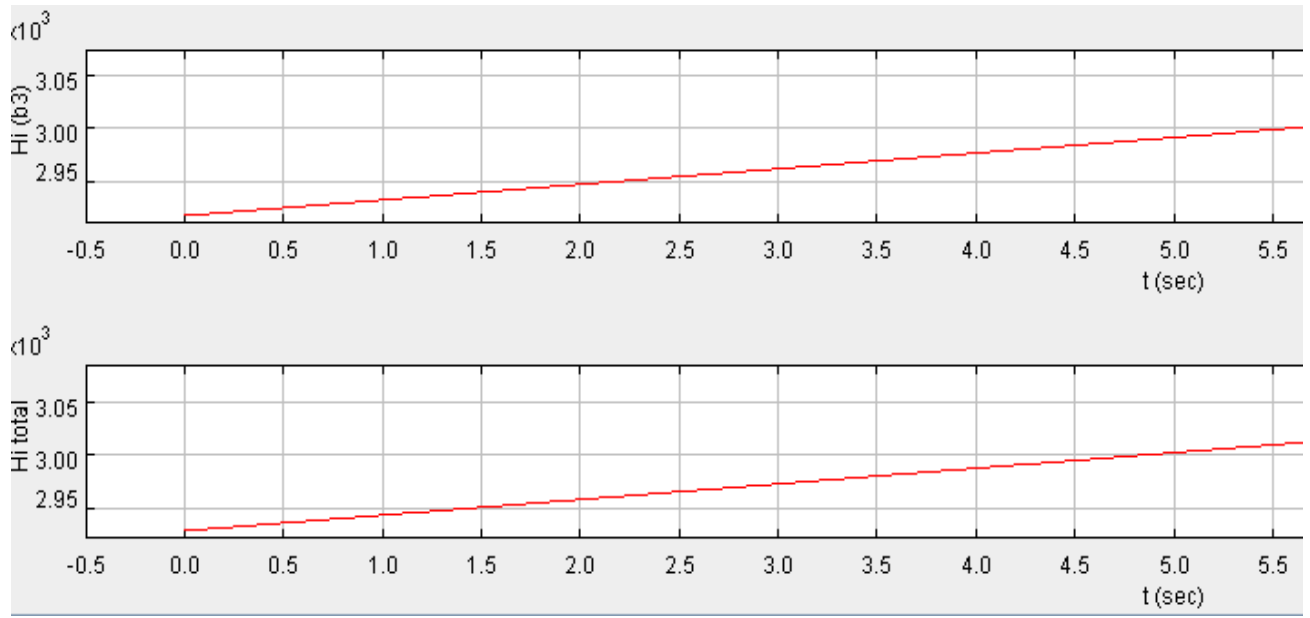

Figure 3: Stabilization of Angular Momentum.
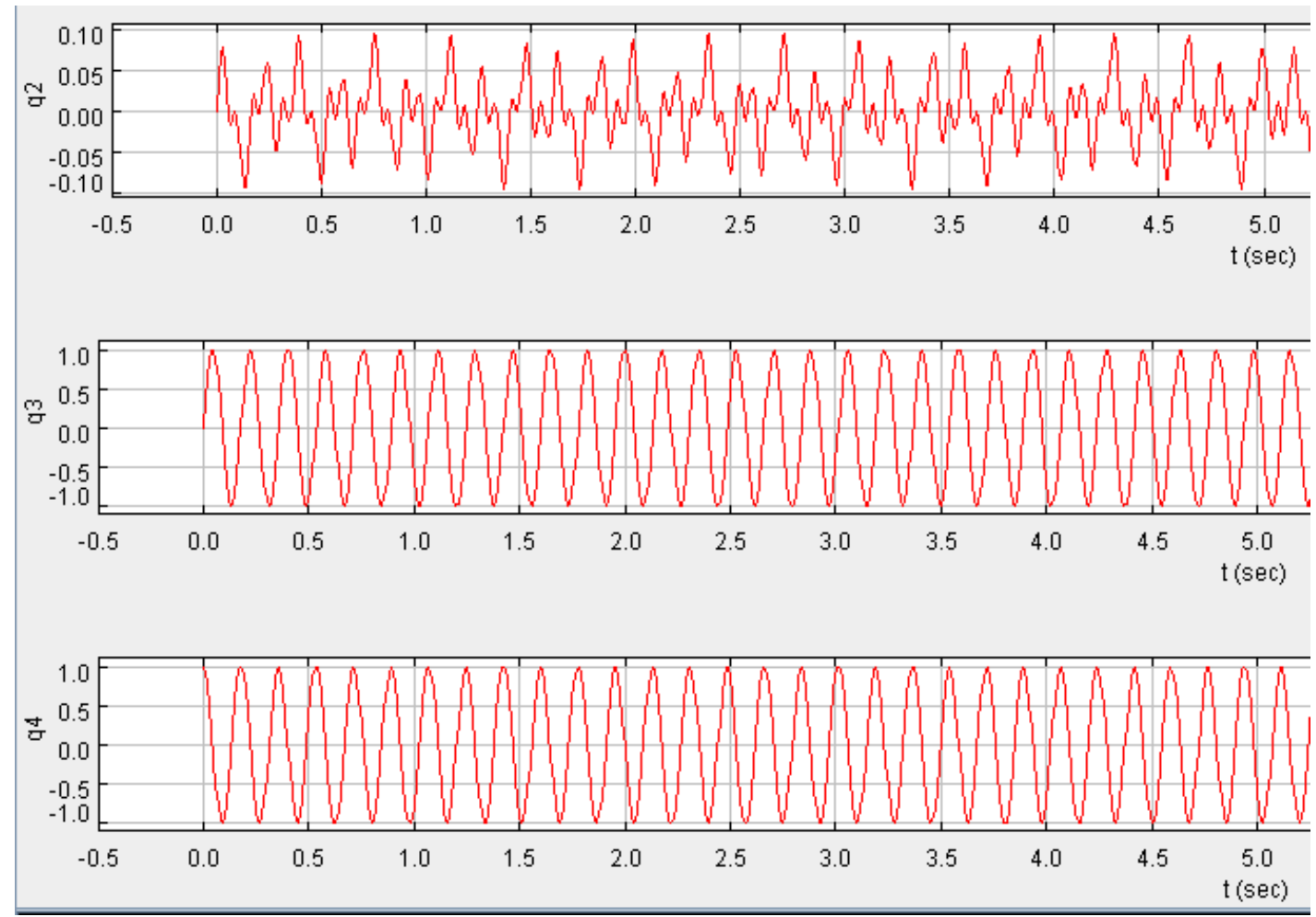

Figure 4: Simulated Quaternion Data. 


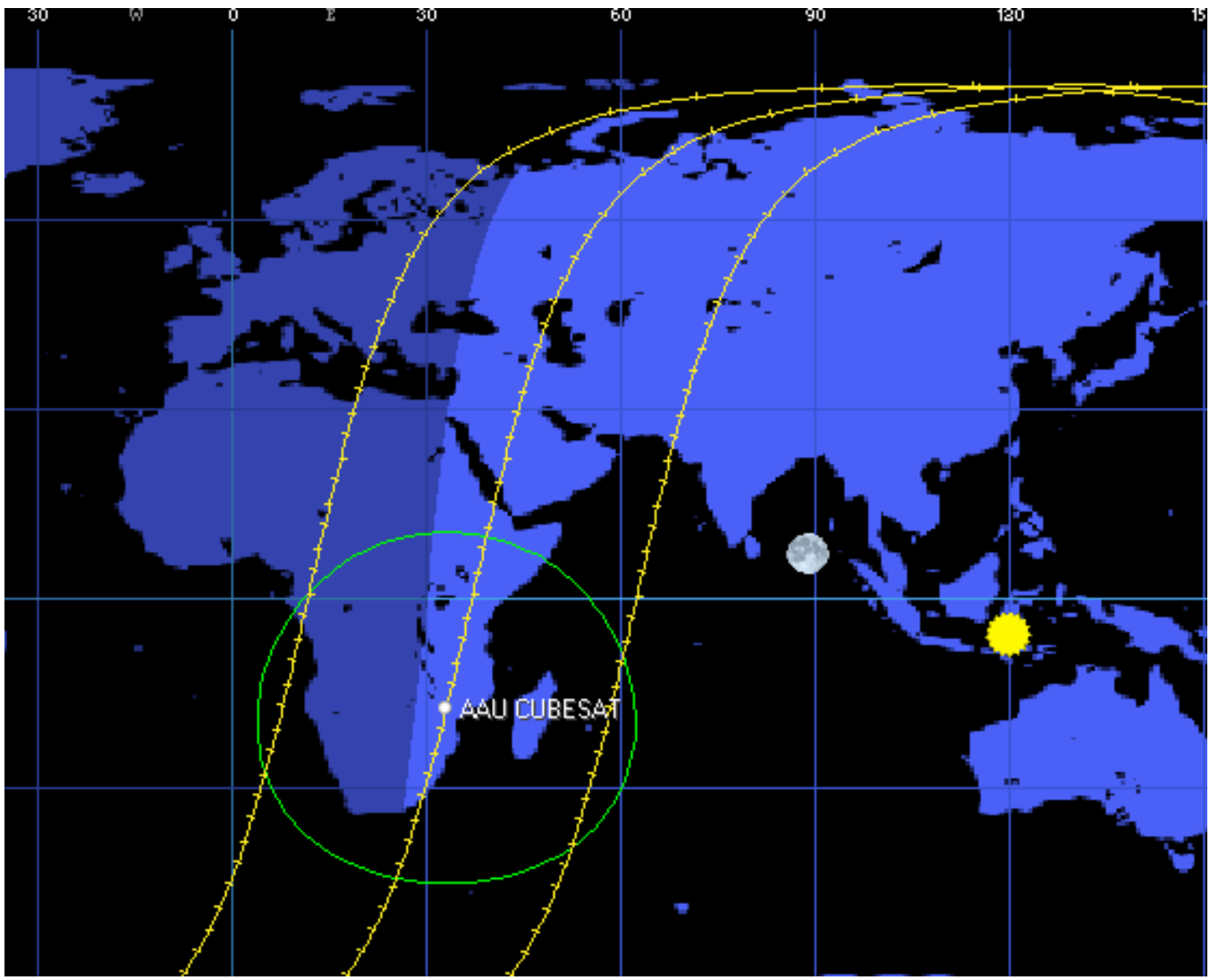

Figure 5: Orbitron Tracking Software.

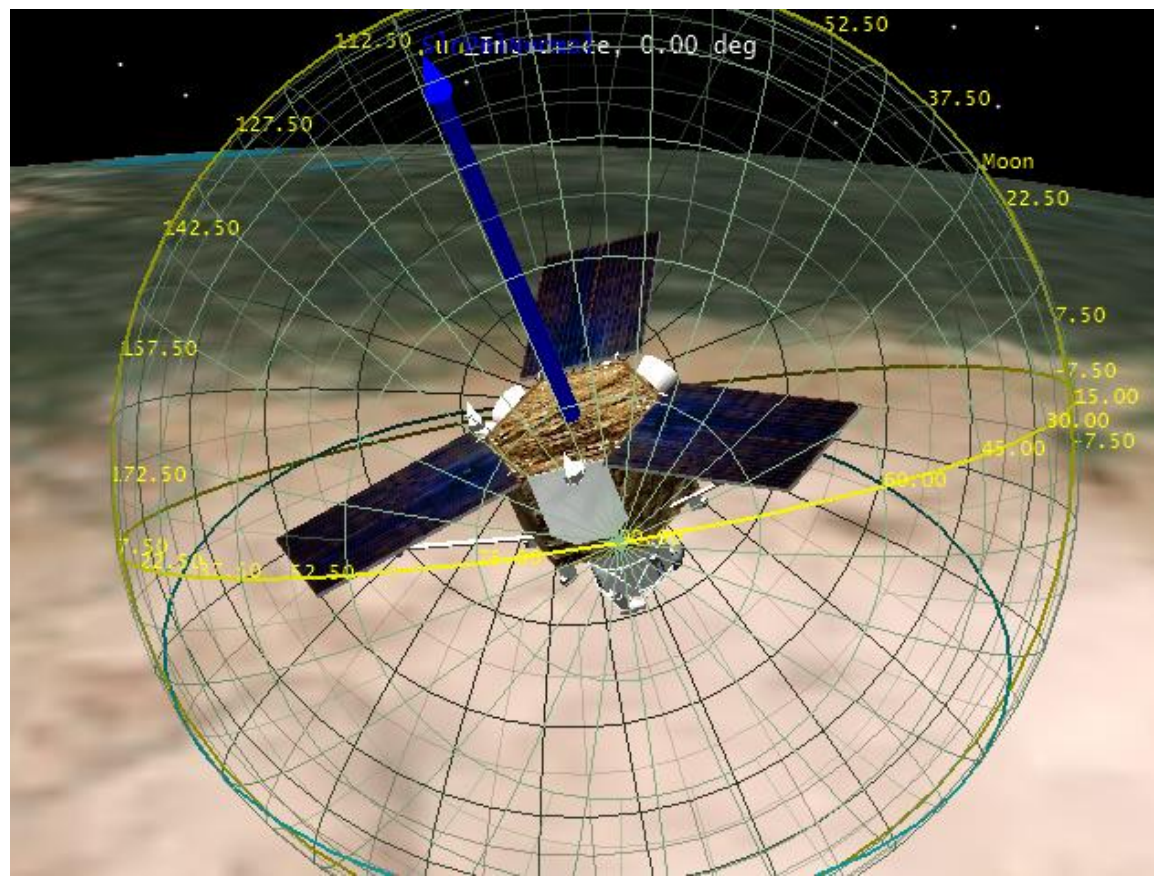

Figure 6: Attitude of the Ship in Orbit, Simulated with AGI Software. 


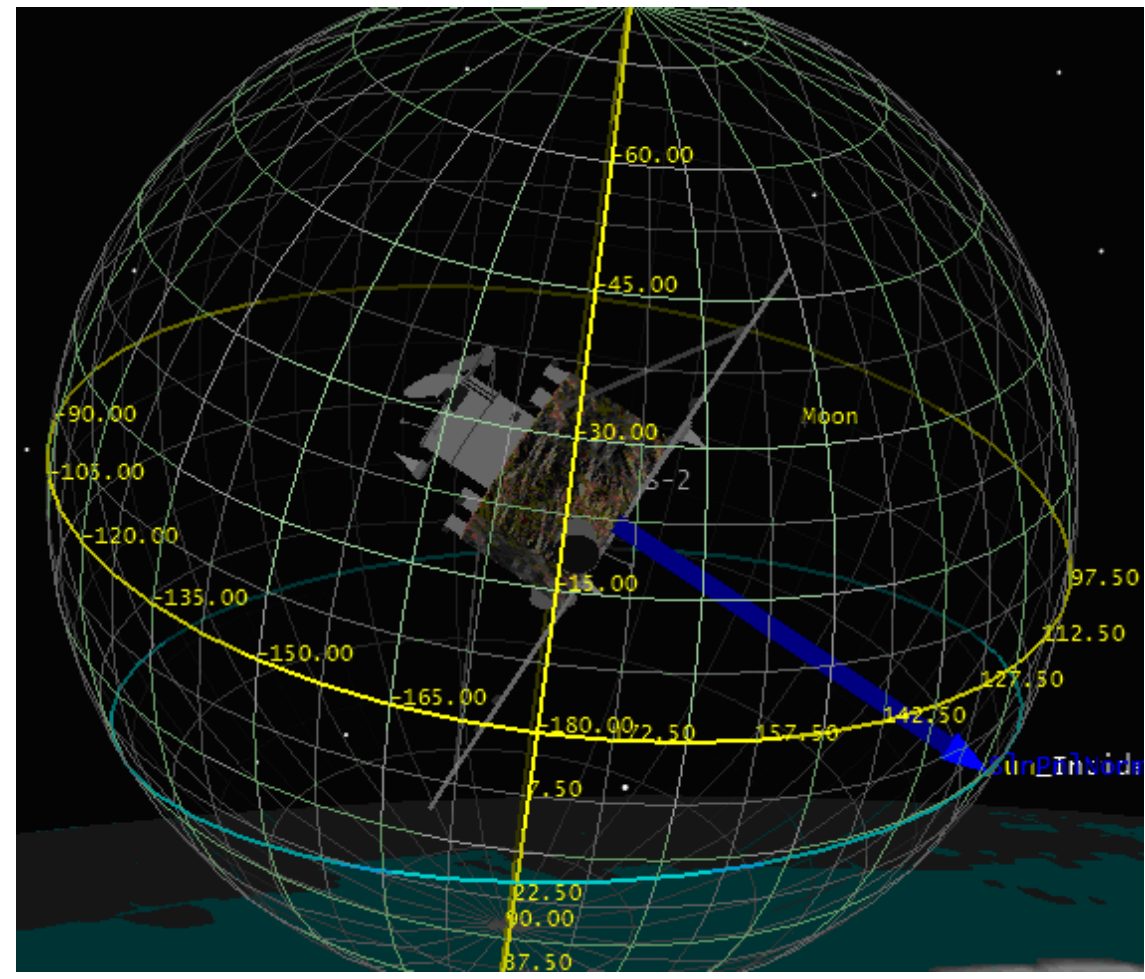

Figure 7: Movement of the Ship to Make a Communication Connection with the Ground Station.

\section{CONCLUSIONS}

For the project, an ADACS system based on triaxial gyroscopes is proposed because they offer a fast stabilization of the $\mathrm{H}$ moment and better results for the quaternion data.

The offer of control mechanisms and attitude acquisition on the market is in line with the project budget.

The project's weight and size limitations are met, as well as its low data memory on the main pc onboard:

- The best results in an angle of vision are for the site of coordinates corresponding to the terrace of the seat of the Faculty of Engineering of the district university, since it offers a horizon with fewer obstacles of direct vision, besides, the result of $47^{\circ} 51^{\prime} 54^{\circ} 36^{\prime}$ allows theoretically to obtain a clearer satellite link at the moment of locating the satellite in the horizon.

- The actuator that fits best to the project because of its weight and better response in time is the gyroscope, an element that is classified as a passive actuator. The response curves shown in Figures 3, 4, 5 show the temporal stability of the satellite axis nutation, the correctable drift of the spacecraft angular momentum, and the quaternions.

- The time of view from the Bogota ground station has a range between 12.8 and $15.30 \mathrm{~min}$, but this can vary to improve the time, according to the launch parameters given by the company in charge of the launch, which until the moment of the simulation are only theoretical and the most adjusted to the needs of the project.

- The satellite orientation simulation results present a lot of information if the STK program of the AGI development house is used, although it was provided for academic purposes for this project, some active actuator modules cannot be developed with the academic version, however, the graphics and the simulation in temporal variation is of very 
good resolution, besides showing the evolution of the axes of the CCS, BCS, OBS, RCS as shown in figures 6,7, 8 .

- The SPG4 software implemented for this work yields high-quality data when compared to two-element line data on NASA/NRAD published data and data displayed by Winorbit, Nova for Windows satellite tracking software.

The simulation results of quaternions, as well as those of angular moments of the vehicle under study, can be carried out with good approximation with the software Jat32 Spacecraft Attitude Simulator since it offers an interesting game with the amounts of angular speed as well as in the main moments of inertia of the Cubesat.

\section{ACKNOWLEDGMENTS}

The authors would like to thank the Universidad Distrital Francisco José de Caldas and the GITEM research group that supported the development and testing of the project.

\section{REFERENCES}

1. R.C. OLSEN, Introduction to the space environment, Naval Postgraduate School, 2003.

A. P. Marins, Determinacao e manobras autonomas de orbitas de satellites artificias en tem-po real usando medidas $P S$ de una frecuencia. Instituto nacional de pesquisas espacias, San José Dos Campos Brasil, pp 139_158, 187_1942000.

2. L. Mendoza, Sistemas coordenados, Ecosat, Mexico, 2007.

3. L. Mendoza, Órbitas keplerianas, Ecosat, Mexico, pp 53-69, 2007

4. J. Bao Yen Tsui, Fundamentals of global positioning system receivers. Second edition, Willey editions, Canada, 2005, pp51-65.

A. Leick. GPS satellite surveying, Willey Interscience, 1995, pp 16-41, pp451-476.

5. D Roddy, Satellite communications. Third edition, New York, 2001, pp21-62, pp67-86.

6. P.J.G. Teunissen. GPS for Geodesy. Second edition, Springer, Berlin, 1998, pp12-36, pp43-106.

A. P. Marins, Determinacao e manobras autonomas de orbitas de satellites artificias en tempo real usando medidas PS de una frecuencia. Instituto nacional de pesquisas espacias, San José Dos Campos Brasil, pp 139_158, 187_1942000.

7. L. Mendoza, Sistemas coordenados. Ecosat, Mexico, 2007.

8. L. Mendoza, Órbitas keplerianas, Ecosat, Mexico, pp 53-69, 2007

9. J. Bao Yen Tsui, Fundamentals of global positioning system receivers. Second edition, Willey editions, Canada, 2005, pp51-65.

A. Leick. GPS satellite surveying, Willey Interscience, 1995, pp 16-41, pp451-476.

10. D. Roddy, Satellite communications. Third edition, New York, 2001, pp21-62, pp67-86.

11. P.J.G. Teunissen., GPS for Geodesy. Second edition, Springer, Berlin, 1998, pp12-36, pp43-106.

12. Heidt, H., Puig-Suari, J., Moore, A.S., Nakasuka, S., Twiggs, R.J., CubeSat: A New Generation of Picosatellite for Education and Industry Low-Cost Space Experimentation, Proceedings of the Utah State University Small Satellite Conference, Logan, UT, August 2001, pp. 1-2, 6.

13. Nason, I., Creedon, M., Puig-Suari, J., CubeSat Design Specifications Document, Revision V, Nov. 2001, pp. 1-6. http://ssdl.stanford.edu/cubesat.

14. Nason, I., Puig-Suari, J., Twiggs, R.J., Development of a Family of Picosatellite Deployers Based on the CubeSat Standard, Proceedings of the IEEE Conference, Big Sky Montana, IEEE, 2002, pp. 1-3. 
15. Boeing Company Space and Communications Group, Delta II Payload Planners Guide. http://www.boeing.com/defensespace/space/delta/delta2/guide/delta2.pdf.

16. Orbital Sciences Corporation, Pegasus User's Guide, Release 5, Aug. 2000. http://www.orbital.com/LaunchVehicles/Pegasus/peg-user-guide.pdf.

17. NASA Johnson Space Center, Space Shuttle Program Payload Bay Payload User's Guide, NSTS 21492, December 2000.NSTS 
